\title{
APLICACIÓN DEL ENFOQUE DE PROMOCIÓN DE LA SALUd EN LA REgión CENTRAL DE OCCIDENTE DE COSTA RICA: LOS PRINCIPALES HALLAZGOS
}

\author{
APPLYING OF THE HEALTH PROMOTION APPROACH IN THE WEST CENTRAL REGION OF \\ COSTA RICA: THE MAIN FINDINGS
}

\author{
Por: MSc. Lidia Isabel Picado Herrera* \\ MSc. Ana Cristina Quesada Monge**
}

* LIDIA ISABEL PICADO HERRERA

Encargada de Mercadotecnia de la Salud, Región Central Occidente, Ministerio de Salud. (lpicadoherrera@ gmail.com)

** MSC. ANA CRISTINA QUESADA MONGE

Docente y coordinadora de la Carrera de Trabajo Social, Sede de Occidente, Universidad de Costa Rica. (cristina. quesada@ucr.ac.cr)
Recibido: 1 agosto 2012. Aprobado: 7 marzo 2013.

\section{RESUMEN}

El enfoque en Promoción de la Salud es un eje técnico-político que busca brindar los medios y las herramientas necesarias a los actores sociales, para que ejerzan un mayor control sobre los determinantes que favorecen la salud. En el caso particular de la Región Central de Occidente de Costa Rica, se han desarrollado una serie de esfuerzos institucionales y comunales para posicionar este paradigma en el abordaje de la salud, en procura de reconstruir una cultura de salud desde una visión positiva.

No obstante, se carecen de investigaciones que permitan conocer la situación real de su aplicación, en instituciones públicas, de la región citada. En este marco, surge la inquietud de indagar al respecto, con el propósito no solo de reflejar la realidad, sino también de aportar insumos que contribuyan a fortalecer la toma de decisiones, por parte de las respectivas autoridades de las instituciones objeto de estudio.

El proceso de investigación evaluación utilizó un enfoque de estudio cualitativo, que permitió un acercamiento más integrador del objeto de estudio, al aplicar dos métodos de investigación evaluativa: investigación-exploratoria e investigación-acción.

El artículo hace un recuento del proceso de investigación evaluativa realizada, donde integra los referentes teóricos-metodológicos utilizados, los principales hallazgos, las conclusiones y algunas recomendaciones generales derivadas de la investigación.

Palabras claves: Salud, enfoque de promoción de la salud, servicios de salud, investigación evaluativa.
Artículo de Investigación científica y Tecnológica según clasificación de Colciencias. 


\section{ABSTRACT}

Focus on Health Promotion is a technical-political axis that seeks to provide the means and necessary tools to social actors, to exercise greater control over the determinants that promote health. In the particular case of the West Central Region of Costa Rica, it has developed a series of institutional and community efforts to position this paradigm health approach, seeking to contribute to better conditions and therefore an optimum quality of life for it population.

However, there is no previous research to let us know the real situation of its application in the public institutions of the region already mentioned. In this framework, there is a concern about the purpose not only of reflecting reality, but also providing input to help strengthening decision making, by the respective authorities of the institutions for study.

The research process used a qualitative research approach, which allowed a more integrative research of the studied object, applying evaluative research methods: Exploratory research and Action research. This is how this article gives a review of the process of evaluation research conducted on the theme "Application of Focus on Health Promotion in the West Central Region of Costa Rica". Also, this article explains the theoretical and methodological framework we used, the conclusions and some general recommendations.

Keywords: Health, Health Promotion Focus, Health services, evaluative research.

\section{FUNDAMENTOS TEÓRICOS DE LA INVESTIGACIÓN EVALUATIVA}

Como primer apartado de este artículo se explica los fundamentos teóricos que orientaron la obtención y el análisis de los hallazgos de las categorías de investigación. Se exponen categorías teóricas centrales, tales como: Proceso Salud- Enfermedad, Enfoque de Promoción de la Salud y Servicios Sociales desde el enfoque de interés.

\subsection{Evolución histórica del abordaje de la salud- enfermedad}

El concepto de salud- enfermedad ha sido objeto de estudio e interpretaciones por diversos enfoques teóricos y epistemológicos, el cual, a lo largo de la historia, ha pasado de ser interpretado a la luz de las visiones mágico, religioso, biológico y psicológica a posiciones más de índole social. Para efectos de este artículo, se centrará el estudio en dos posiciones teóricas sobre la saludenfermedad: biológico y sociológica.

Según Carmona, Rozo y Mogollón (2005), el primer enfoque concentra su interés de intervención en el cuerpo. Es decir, el cuerpo es considerado como 
un aparato mecánico y un ente receptor de acciones y sustancias que pueden modificar su estado de salud y enfermedad. En tal sentido, el ser humano es reducido a la suma de órganos, dado que le interesa trabajar solo con las personas que manifiesten problemas físicos. Por tal motivo, este enfoque considera la salud como un estado de equilibrio, mientras que la enfermedad es considerada como desequilibrio y anormalidad.

El enfoque biologísta abandona todo tipo de visiones humanistas en los procesos de intervención y actuación con el ser humano.

A raíz de esta debilidad que presenta el enfoque biologísta, surge en los últimos años, la propuesta de ver la salud desde las posturas sociológicas, la cual está cobrando fuerza e importancia en el contexto actual. Según este enfoque, "la salud no es sencillamente la ausencia de enfermedad, es algo positivo, una actitud alegre hacia la vida y la aceptación entusiasta de la responsabilidad que la vida impone a la persona" (Carmona, et al, 2005: 67).

Esta perspectiva teórica que propone Carmona, et al (2005), tiene grandes coincidencias con la propuesta que establece Breilh (Citado en Bergonzoli, 1994). Este autor concibe la salud como un proceso y no como un estado asignando, dado que las responsabilidades en la construcción de la salud y del mejoramiento de las condiciones de vida, no solo está en las personas de manera individual, sino en la sociedad en su forma de organización colectiva.

En este avance del concepto de salud, es donde se logran visualizar tres escenarios de actuación, los cuales son: salud, riesgo e intervención. De los tres escenarios, el primero es en donde se posiciona la actuación del Enfoque de Promoción de la Salud.

\section{2. ¿Qué se entiende por Promoción de la Salud?}

La Promoción de la Salud es considerada como una función esencial de la salud pública. Constituye un enfoque primordial en el abordaje de los determinantes del proceso salud-enfermedad, específicamente en cuanto a potenciar y desarrollar factores protectores y condiciones salutogénicos en la población. Es decir, actuar fortaleciendo el escenario de salud. Ello se refleja en la definición establecida en la Carta de Otawa, la cual dice: "La Promoción de la Salud es proporcionar a los pueblos los medios necesarios para mejorar su salud y ejercer un mayor control sobre la misma. Trasciende la idea de 
formas de vidas sanas, para incluir las condiciones y requisitos para la salud que son: paz, vivienda, educación, alimentación, renta, ecosistema estable, recursos sostenibles, justicia social y equidad". (1986, citada por la Dirección General de Salud, Ministerio de Salud, 2006: 9).

Cinco líneas de acción caracterizan este enfoque: generación de políticas públicas saludables, creación de ambientes saludables, fortalecimiento de la participación social, desarrollo de potencialidades individuales y colectivas y reorientación de servicios de salud.

\subsection{Servicios sociales desde el enfoque de la Promoción de la Salud}

Para efectos de la investigación evaluativa desarrollada, se entendió como servicio social en el ámbito de la salud, todo plan, programa, proyecto o actividad institucional que se dirija a fomentar, específicamente el enfoque de Promoción de la Salud.

Para Nidia Morera, los servicios sociales se pueden concebir como "toda actuación generada dentro de la sociedad, a través de una institución pública y privada, dirigida a la satisfacción de necesidades de personas o grupos, con el objetivo de lograr la satisfacción de necesidades básicas y su desenvolvimiento dentro de la sociedad en la que se encuentra inmersa". (2001: 19).

Un elemento que destaca esta autora sobre el tema de los servicios sociales, es que los mismos, a lo largo de la historia, han ido transformando su enfoque de actuación en la sociedad. Es decir, "han ido pasando de la satisfacción de necesidades vitales a la satisfacción de necesidades culturales". (Morera, 2001: 19) Es por eso, que hoy en día se pueden observar servicios sociales orientados a la atención o prevención de problemáticas específicas, como a la promoción de condiciones de vida de las personas y comunidades.

Tomando en consideración lo antes expuesto, se puede decir que los servicios sociales derivados de un enfoque de Promoción de la Salud son un tipo de servicio social ampliado, es decir un servicio social dirigido al conjunto de la población, de acuerdo a los principios de universalidad, que trasciende las acciones de carácter asistencial, e integra acciones de promoción y desarrollo a nivel individual, familiar, grupal y comunal. Son servicios sociales que facilitan las condiciones objetivas y elementales que permiten mejorar la calidad de vida del conjunto de la ciudadanía (Morera, 2001: 31-32). 
Al considerar esta distinción de cómo se manifiestan los servicios sociales bajo un enfoque de Promoción de la Salud, las autoras de este artículo, efectuaron un proceso de investigación evaluativa de los servicios sociales de las instituciones públicas de la Región Central Occidental de Costa Rica, desde este enfoque, se considera la evaluación de servicios sociales como:

“...una investigación aplicada, de carácter sistemático, que pretende obtener información significativa sobre la planificación, el desarrollo y los resultados de un determinado servicio o programa social, con el objetivo de valorar el grado de adecuación de este a la previsiones iniciales y establecer unos juicios de valor (basados en análisis comparativo de aquello o que se ha conseguido con aquello que se pretendía conseguir); deben facilitar los procesos de toma de decisiones, tomando siempre en cuenta, la utilidad social de las conclusiones, de tal manera que estas permitan mejorar y optimizar el mejoramiento y los resultados de los servicios y programas evaluados. (Gómez, 2003: 57)

Considerando tal definición, se propuso alcanzar el siguiente objetivo general: "Evaluar la integración del enfoque de promoción de la salud y la capacidad instalada de las instituciones públicas involucradas en la producción de procesos de salud de la Región Central Occidental de Costa Rica, para contribuir al fortalecimiento de este componente como función esencial de la Salud Pública".

\section{MÉTODO Y ENFOQUE DE LA INVESTIGACIÓN EVALUATIVA REALIZADA}

Los métodos base de la investigación evaluativa fueron dos: "investigaciónexploratoria" e "investigación-acción".

El primer método se realizó con base en una serie de actividades de investigación de enfoque cualitativo, que permitió el acercamiento a tres tipos de actores sociales de las instituciones públicas de la región, a saber: Jefaturas Regionales, Jefaturas Locales y miembros de los Equipos Técnicos. Además se logró tener acceso a fuentes de información documental, que permitió registrar y analizar la actuación de las instituciones presentes en la región.

Con dicho método se logró obtener la percepción que tienen estos tres actores sociales sobre los enfoques de abordaje de la salud, la capacidad instalada que cuentan las instituciones para hacer efectivo estos servicios y el perfil de aptitudes 
que caracterizan al personal encargado de desarrollar la Promoción de la Salud. Además se realizó un proceso de investigación-acción con un proyecto de extensión docente, que complementó las acciones evaluativas. Por medio de este proyecto se logró reflexionar con un grupo de funcionarios de instituciones públicas de la región, sobre: el marco normativo-legal que fomenta a las instituciones trabajar el enfoque de Promoción de la Salud, las posiciones teóricas sobre el proceso de salud-enfermedad, los diversos enfoques de actuación sobre ese proceso, las propuestas teóricas y metodológicas para formular y evaluar proyectos a partir del enfoque en estudio. También se registraron las etapas metodológicas de formulación, ejecución y evaluación de proyectos específicos con enfoque de Promoción de la Salud en la región.

\section{PRINCIPALES HALLAZGOS DE LA INVESTIGACIÓN EVALUATIVA}

\subsection{Marco legal costarricense una oportunidad para implementar la Promoción de la Salud}

El marco legal costarricense, en lo concerniente a la Constitución Política y las leyes- específicamente de Salud, Educación, Código de Niñez y Adolescencia, la Ley de Creación del Instituto Costarricense del Deporte y la Recreación (ICODER), Código Municipal- si bien el espíritu de las mismas no están orientados, ni destacan, el enfoque de Promoción de la Salud como tal, si consideran de manera implícita, componentes, factores protectores, ejes temáticos, líneas de acción, entre otros, asociados al mismo, como son la generación de ambientes físicos y sicológicos saludables, reorientación de servicios de salud, desarrollo de potencialidades individuales y colectiva, generación de políticas públicas saludables que constituyen condiciones y oportunidades para justificar desde este marco, acciones de Promoción de la Salud (PS).

En cuanto a los decretos emitidos en Costa Rica, resulta también significativo el hecho de que la mayoría de ellos no parten del enunciado de PS, pese a que los mismos forman parte de la lista de decretos considerados dentro del marco legal de PS.

Las acciones parten de factores protectores, su fundamentación y objetivos se orientan hacia grupos de riesgos para la prevención de enfermedades, especialmente cardiovasculares, asimismo del tabaco y la violencia. De este modo, las actividades predominantes son alimentación saludable, recreación, actividad física, deporte, es decir, implica por consiguiente el abordaje de los 
factores de riesgo a partir del mejoramiento de los estilos de vida saludable. La excepción en este caso la constituye el Decreto N 31516-S 12/12/2003 "Reglamento de la Promoción de la Salud", pues no sólo crea la Comisión Nacional de PS, sino que reglamenta su funcionamiento. Representa un logro para el país en cuanto da cumplimento a los compromisos internacionales y define procesos de planificación, investigación y evaluación de la PS, así como su fortalecimiento, mediante la participación social y las alianzas. Por otra parte, busca incidir en la definición de políticas, leyes y normas en esta materia, con lo cual se asegura en parte, su continuidad y sostenibilidad.

\subsection{Promoción de la Salud en el marco de los planes nacionales e institucionales en Costa Rica}

Los planes nacionales representan el fundamento técnico y político que guía las actuaciones de las diversas instituciones, en este caso, los afines a la generación de salud. A continuación se mencionan los contenidos más relevantes relacionados con Promoción de la Salud, identificados en:

\section{Cuadro \# 1. Análisis de los planes nacionales e institucionales en Costa Rica}

\begin{tabular}{|c|c|}
\hline PLANES & CONTENIDO \\
\hline $\begin{array}{l}\text { PLANES NACIO- } \\
\text { NALES DE DESAR- } \\
\text { ROLLO 2006-2010 }\end{array}$ & $\begin{array}{l}\text { Este Plan Nacional de Desarrollo se caracteriza por la impor- } \\
\text { tancia que da al desarrollo de la actividad física, la recreación, } \\
\text { el deporte. De igual manera, se consideran proyectos dinam- } \\
\text { izadores y articuladores que propicien el desarrollo cultural } \\
\text { comunitario y la capacidad de gestión cultural de los actores } \\
\text { locales, con énfasis en los jóvenes. Otro aspecto a destacar } \\
\text { es el fomento de los valores identitarios y la creatividad en } \\
\text { la población joven atendida por el Ministerio de Educación } \\
\text { Pública (MEP), para el logro de un desarrollo integral, el cual } \\
\text { hasta hace poco fue un campo invisibilizado, pese a ser un } \\
\text { factor favorecedor de la salud, pues permite a las personas } \\
\text { desarrollarse en esta área en especial a quienes tienen talento, } \\
\text { proporcionando satisfacción y realización. } \\
\text { No obstante, este Plan tiene como punto de partida la iden- } \\
\text { tificación de una serie de carencias y problemas sobre los } \\
\text { cuales se orientan las acciones planteadas para dar respuesta } \\
\text { a los mismos. Algunos de los ejes establecidos son la lucha } \\
\text { contra la pobreza, la lucha contra las drogas, prevención de } \\
\text { la violencia, entre otras, que requieren de un abordaje inter- } \\
\text { sectorial, así como de una clara definición de las rectorías } \\
\text { según competencia y ámbito de acción. }\end{array}$ \\
\hline
\end{tabular}




\begin{tabular}{|c|c|}
\hline $\begin{array}{l}\text { PLAN DE AC- } \\
\text { CIÓN ESTRATEGIA } \\
\text { NACIONAL DE } \\
\text { ALIMENTACIÓN } \\
\text { SALUDABLE, AC- } \\
\text { TIVIDAD FÍSICA } \\
\text { Y SALUD, COSTA } \\
\text { RICA. 2006-2021. } \\
\text { Agosto 2006. }\end{array}$ & $\begin{array}{l}\text { Implementa el "Proyecto } 5 \text { al día" y el Decreto Ejecutivo No } \\
\text { 32886-S-C-MEIC-MTSS-MP-G del año 2006, así como a la } \\
\text { POLÍTICA NACIONAL DE SALUD 2002-2006. Busca fortal- } \\
\text { ecer la educación y comunicación alimentaria nutricional y } \\
\text { la actividad física en la población, mediante un programa de } \\
\text { educación alimentaria nutricional y actividad física para el } \\
\text { consumidor. } \\
\text { Además, delega a las comisiones de seguridad alimentaria y } \\
\text { nutricional (COSAN), la ejecución de proyectos relacionados } \\
\text { con alimentación saludable, actividad física y salud en cen- } \\
\text { tros educativos y en cada uno de los municipios, para lo cual } \\
\text { propone articular las instituciones gubernamentales y organ- } \\
\text { ismos internacionales en el desarrollo de acciones vinculadas } \\
\text { con la estrategia, mediante el desarrollo de un Plan Maestro } \\
\text { de Inversión, así como el desarrollo de talentos. } \\
\text { Pretende lograr la integración de los componentes de activi- } \\
\text { dad física y alimentación saludable en los planes de estudio } \\
\text { de la educación preescolar, primaria y secundaria, lo cual } \\
\text { constituye un gran avance, ya que de alcanzar este objetivo } \\
\text { estaría dando un paso cualitativo hacia el enfoque de PS, tanto } \\
\text { en el contenido al trabajarse desde una perspectiva positiva, } \\
\text { como en la cobertura al incorporar toda la población (niñez } \\
\text { y adolescente cautiva). } \\
\text { Al igual que el Plan Nacional, el propósito de tales acciones, } \\
\text { fundamentalmente, es la prevención de factores de riesgo. }\end{array}$ \\
\hline $\begin{array}{l}\text { PLAN NACIONAL } \\
\text { DE SALUD MEN- } \\
\text { TAL 2004-2010 }\end{array}$ & $\begin{array}{l}\text { Integra aspectos establecidos en el marco legal y políticos } \\
\text { del país, tales como: los Decretos } N^{\circ} 34030 S-M A G-M E I C- \\
\text { MEP "Red } 5 \text { al Día", Decreto Ejecutivo } N^{\circ} 32886-S-C-M E I C- \\
\text { MTSS-MP-G del año } 2006 \text { (RECAFIS) y Código de Niñez y } \\
\text { Adolescencia. } \\
\text { Entre los aspectos más relevantes destacan: aumentar el } \\
\text { consumo de frutas y vegetales en todos los grupos de edad } \\
\text { y actividad físicas, recreativas, entre otros. } \\
\text { Define un abordaje integral de la salud, en donde se resalta } \\
\text { la PS, como una de las acciones a desarrollar para mantener } \\
\text { la salud mental. Retoma el concepto y líneas de acción de la } \\
\text { Carta de Ottawa. } \\
\text { La promoción de la salud mental incluye acciones dirigidas } \\
\text { a modificar los grandes determinantes biológicos, políticos, } \\
\text { sociales, educativos, culturales y económicos. Se debe educar } \\
\text { para la incorporación de estilos de vida saludables en todos los } \\
\text { aspectos de la vida humana, tales como el disfrute de la sexu- } \\
\text { alidad y reducción de embarazos no deseados, autocuidado de } \\
\text { la salud, alimentación saludable, educación para la utilización } \\
\text { del juego y actividades recreativas y deportivas, vigilancia y } \\
\text { estimulación del desarrollo sicomotor infantil, importancia } \\
\text { de las relaciones interpersonales y familiares armoniosas y } \\
\text { consejería genética para evitar enfermedades mentales he- } \\
\text { reditarias y malformaciones congénitas del sistema nervioso, } \\
\text { programas educativos para reducir los traumas del parto e } \\
\text { inmunizaciones oportunas. }\end{array}$ \\
\hline
\end{tabular}




\begin{tabular}{|c|c|}
\hline $\begin{array}{l}\text { PLAN ESTRATÉ- } \\
\text { GICO DE RECAFIS }\end{array}$ & $\begin{array}{l}\text { Es consistente con el marco legal costarricense. Específica- } \\
\text { mente con los siguientes decretos y leyes: Decreto Ejecutivo N }{ }^{\circ} \\
32886-S-C-M E I C-M T S S-M P-G \text { del año 2006, Política Nacio- } \\
\text { nal 2002-2006, Código de Niñez y Adolescencia, entre otros; } \\
\text { destaca la importancia de incorporar aspectos relacionados } \\
\text { con alimentación saludable, actividad física y recreativas. } \\
\text { Por otra parte evidencia las políticas nacionales en actividad } \\
\text { física, deporte y recreación y salud, siendo esta una de las } \\
\text { líneas de acción de PS, también plantea el establecimiento de } \\
\text { alianzas y acciones intersectoriales, vinculándose con otra } \\
\text { de las líneas de acción, como lo es el fortalecimiento de la } \\
\text { Participación Social. Otro elemento, es el relacionado con la } \\
\text { formación de talento humano, ya que promueve la incor- } \\
\text { poración de esta temática en los currículos de formación } \\
\text { profesional de las universidades en el país. }\end{array}$ \\
\hline $\begin{array}{l}\text { PLAN NACIONAL } \\
\text { DE EDUCACIÓN } \\
2006-2015\end{array}$ & $\begin{array}{l}\text { La educación representa un eje fundamental no solo desde el } \\
\text { punto de vista académico, sino también para la formación } \\
\text { y desarrollo humano y por ende, un factor favorecedor de } \\
\text { la salud. Por ello aunque no se dimensione la PS como tal, } \\
\text { desde la misión, las metas plantean acciones que permiten } \\
\text { identificar el escenario educativo, como un campo de enorme } \\
\text { potencial para la implementación del enfoque de PS, desde sus } \\
\text { líneas de acción, tales como la creación de ambientes físicos y } \\
\text { sicológicos saludables, protección y convivencia en armonía } \\
\text { con la naturaleza, desarrollo de potencialidades individuales } \\
\text { y colectivas saludables, de igual manera talentos, habilidades, } \\
\text { creatividad, comportamientos y actitudes emprendedoras, } \\
\text { convivencia pacífica. } \\
\text { Por otra parte, para fortalecer procesos de participación social } \\
\text { en las gestiones de este ámbito, además se pueden fomentar } \\
\text { actividades que favorezcan estilos de vida saludable en toda } \\
\text { la población estudiantil y comunidades educativas en general. } \\
\text { Interesante resulta también el hecho de que se integre un } \\
\text { plan para mejorar la capacidad productiva y emprendedora } \\
\text { que representa posibilidades para la generación de ingresos } \\
\text { económicos, otro de los factores básicos en marco de la PS y } \\
\text { calidad de vida. } \\
\text { En términos generales se observa consistencia teórica, no solo } \\
\text { con el PND sino también, con los decretos considerados como } \\
\text { afines a la PS, analizados en esta investigación pues integra } \\
\text { prioridades definidas. }\end{array}$ \\
\hline
\end{tabular}

Fuente: Elaboración propia a partir del informe final de Picado L. \& Quesada A. (2012).

Como se puede apreciar en el cuadro anterior, los planes nacionales, en términos generales, incorporan ejes y acciones dirigidas al fomento de Estilos de Vida Saludable, vinculados a su vez con líneas de acción del enfoque de Promoción de la Salud. Sin embargo, dichos planes están fundamentados desde una óptica preventiva, porque su propósito es actuar sobre factores de riesgo de enfermedades cardiovasculares, violencia, obesidad, enfermedades mentales, embarazos, entre otros. 


\subsection{Promoción de la Salud en la Región Central de Occidente de Costa Rica}

A nivel regional, específicamente en la Región Central de Occidente, ámbito geográfico de la investigación evaluativa ejecutada, existe el Plan Estratégico Regional Intersectorial de Promoción de la Salud 2008-2011, el cual integra una serie de acciones dirigidas al fomento de este enfoque en dicha región. Impulsadas de manera conjunto por diversas instituciones, tales como: Ministerio de Salud, Universidad de Costa Rica, Sede de Occidente, Ministerio de Educación, Dirección Regional Educativa de Occidente, Caja Costarricense del Seguro Social, Región Central Norte, Acueductos y Alcantarillados, Federación Occidental de Municipalidades de Alajuela, Instituto Costarricense del Deporte y la Recreación.

En este plan, las instituciones públicas de la Región Central de Occidente, desarrollaron un proceso de planificación regional, que incorpora proyectos y estrategias orientadas a direccionar y darle mayor sostenibilidad a la ejecución de las acciones de Promoción de la Salud.

Entre los aspectos más relevantes considerados en el mismo, se encuentran:

- Capacitación de actores sociales internos y externos

- Planificación, seguimientos y evaluación de las acciones de PS

- Estrategias de comunicación de programas, proyectos e iniciativas de promoción de la salud en la Región Central de Occidente (RCO)

- Fomento de acciones técnicos-políticos y financieros de las autoridades institucionales internacionales, nacionales, regionales y locales para fomentar procesos de Promoción de la Salud

- Impulso de actividades educativas e intercambio de experiencias de acciones y/ o proyectos implementados con enfoque de Promoción de la Salud de la RCO.

- Investigación. (Comisión Regional Intersectorial de Promoción de la Salud, 2008).

Una vez identificado las oportunidades y limitaciones presentes en los marcos normativos nacionales e institucionales referentes al tema de Promoción de la Salud, el proceso de investigación se dirigió a conocer la percepción y opinión que tenían los funcionarios y funcionarias de diversas instituciones públicas sobre este tema. A continuación se presenta los principales resultados sobre esta investigación.

Palobra No. 13. Agosto de 2013 


\subsection{Esfuerzos por diferenciar los enfoques de actuación en la salud, pero con predominio en la prevención y atención de la enfermedad}

Un hallazgo que se obtuvo a la hora de entrevistar a los grupos participantes de la investigación, sobre el concepto de Promoción de la Salud, Prevención y Atención de la Enfermedad, fue que hubo más dificultad en definir el primer enfoque que los subsiguientes.

Por ejemplo, en cuanto a la definición de Promoción de la Salud, sus respuestas se centraron en la identificación de factores protectores de la salud, tal como se ejemplifica a continuación:

\footnotetext{
"Es un enfoque donde se pretende incentivar a la población a tener estilos de vida saludable y a que la gente desarrolle un marco integral como persona y crecer en un entorno positivo (social, física, entre otros)" (MS);

"Toda actividad que se realiza para mejorar el estilo de vida (ICODER)";

"Estar sano, mantengámonos sanos." (UCR).

"Son las acciones que se realizan para promocionar estilos de vida saludable, y lo que es actividad física, recreación y todo lo que nos va a llevar a precisamente en un bienestar en la salud de las personas o factores que favorecen la salud" (MS).
}

Estas respuestas se asocian más a la tendencia de centrar este enfoque con estilos de vida saludables, donde reflejan un accionar limitado, puesto que se circunscribe a ciertos grupos y ejes temáticos, especialmente relacionado con actividad física y alimentación, dejando de lado ejes sustantivos como los son las políticas públicas, el desarrollo humano, ambientes saludables, entre otros.

En cuanto a la Prevención y Atención de la Enfermedad, los actores describen con más facilidad estos dos enfoques, porque en sus respuestas logran identificar los factores de riesgo y daño. A continuación se citan algunas de las respuestas expuestas por los entrevistados:

Prevención: "Actividades que requieren de una acción de sistematización en actividad física para prevenir enfermedades y mejorar su calidad de vida." (ICODER)

"Va más encaminada a aquellas personas que tienen factores de 
riesgo que ponen en riesgo su salud, obesidad" (MS), "Ejecutar acciones que nos vayan a prevenir problemas sanitarios o de salud en este caso." (MAG).

Atención: "Es cuando ya está establecida una enfermedad, ya se detectó. Hay que dar medidas de protección para que no se siga un proceso o no se lleve al final que sería la muerte de la persona y medidas de control sería que se lleve una dieta adecuada, que se tome los medicamentos"(MS),

"Es algo directo, acceder a un centro que de la atención directa sobre una dolencia sobre una situación que está afectando mi sistema biológico" (MINAET).

Estas respuestas denota el predominio de brindar servicios de salud desde una perspectiva biologista, médico-asistencialista. En este sentido, se aleja de una visión social del abordaje del riesgo y la enfermedad.

Ahora bien, en el proceso de investigación también se indagó cuál de dichos enfoques predominan en sus respectivas instituciones, a lo que respondieron la mayoría, la Prevención de la Enfermedad o Riesgos, hallazgo que confirma que se sigue privilegiando el interés de abordar la salud desde una perspectiva biologista.

Algunas de las expresiones que ejemplifican tales hallazgos son: "Yo quisiera que el MS tuviera más implementado lo que es la PS, porque la promoción está dentro de lo que es la visión del MS, pero lamentablemente no hemos llegado a una verdadera promoción, considero que estamos en lo que es la prevención" (MS).

Mientras que el Ministerio de Agricultura y Ganadería menciona que trabajan con los dos enfoques: "Prevención y promoción. Eso es lo que estamos trabajando en el sector agrícola, estamos tratando que los alimentos sean inocuos, tecnologías limpias y prevención que los productos químicos que se utilicen no sean tóxicos, contaminantes".

Estas expresiones reflejan la necesidad de reforzar procesos de trabajo, que conlleven al efectivo cambio del paradigma para el abordaje de saludenfermedad, desde una visión social. 


\subsection{Contradicciones entre el decir y el hacer}

A partir del análisis documental, así como de las respuestas de la población entrevistada, se encuentran algunas contradicciones entre lo que se plantea en el marco político-gerencial y la realidad existente en cuanto a la operacionalización del enfoque de Promoción de la Salud. A continuación se presenta la situación encontrada al respecto:

3.5.1) Directrices institucionales débiles que estipulan el enfoque de Promoción de la Salud

Algunas de las categorías analizadas en la investigación evaluativa fueron las directrices institucionales, los programas y los proyectos que incorporan el tema de estudio. Al respecto se encontró que solo tres instituciones contienen de manera explícita dicho enfoque.

Una de ellas fue el Ministerio de Salud, que define como política institucional la "mercadotecnia de la estrategia de la promoción de la salud y de la cultura de no exclusión". Así se manifiesta en el siguiente objetivo: "Capacitación y formación de responsables institucionales en destrezas claves para la instrumentalización de la promoción y el desarrollo de la cultura de la no exclusión". (Ministerio de Salud, 2008-2010: 11)

Por otra parte, la Caja Costarricense del Seguro Social (CCSS) en el Plan de Acción de la Gerencia Médica 2007-2012 y el Plan Estrategico Institucional integra algunos objetivos institucionales sobre el enfoque en Promoción de la Salud, a saber: "Propiciar el desarrollo y fortalecimiento de la promoción de la salud y la prevención de la enfermedad, como ejes clave de proceso de atención integral de la salud" (CCSS, 2006: 8) y "Fortalecer la promoción de la salud y la prevención de la enfermedad". En dichos documentos expone las siguientes prioridades de actuación:

"Actualización y ejecución del Plan de Atención de Salud a las Personas (PASP), con un fuerte contenido de acciones preventivas y de promoción de la salud (Acción alternativa), establecimiento de una alianza estratégica con los Ministerios de Salud y Educación, extensiva posteriormente a otras entidades públicas y privadas para promover el desarrollo de una cultura de vida saludable a nivel interno y fortalecimiento de los programas y actividades de recreación y salud dirigidos a los funcionarios". (CCSS, 2006: 64) 
La tercera institución que integra explícitamente una intención por fomentar el enfoque de Promoción de la Salud fue la Universidad de Costa Rica. Entre sus políticas institucionales considera:

“Establecerá vínculos con el sector externo, con el fin de promover el desarrollo, a escala nacional, de una cultura de respeto por el ambiente y una vida saludable.

Estimulará la creación y el fortalecimiento de los espacios necesarios que promuevan la realización de actividades recreativas, deportivas y artísticas" (Picado L \& Quesada, 2012: 52)

Específicamente, en la Sede de Occidente de la Universidad de Costa Rica, establece en su Plan Estratégico para el año 2010-2014, algunas áreas que integra el Enfoque de Promoción de la Salud, tales como: "Deportivos, recreativos y artístico - cultural. La promoción, el apoyo y el estímulo de la práctica individual y colectiva del deporte y la recreación de los habitantes de la República, componente fundamental para la salud integral de la población".

Para operacionalizar tales áreas estratégicas, se definen como proyectos específicos: Para Vivir Mejor: Encuentros de Promoción de la Salud, Promoción de Estilos de Vida Saludables, Instalaciones Deportivas y Recreativas de la Sede de Occidente.

El resto de instituciones investigadas, a pesar de tener una normativa nacional que los involucra en el fomento de la Promoción de la Salud, no incorporan de manera explícita acciones a favor de este enfoque, en sus planes y proyectos institucionales. No obstante, integran ejes y objetivos que constituyen una oportunidad para abordar factores protectores en las áreas, tales como: ambientales, estilos de vida saludables, potencialidades individuales y colectivas. A continuación se destacan algunos de ellos:

El MEP, por ejemplo, en el Plan Nacional de Educación, que es retomado en el Plan Operativo Regional, establece los siguientes objetivos: "Fortalecer el aprendizaje para la protección y convivencia en armonía con la naturaleza, comprendiendo el carácter de interdependencia, sostenibilidad y respeto a toda forma de vida". (Picado L \& Quesada A, 2012: 13) 
Por su parte, estos lineamientos se operacionalizan con los siguientes programas:

- Fortalecimiento de la formación ética, estética y ciudadanía.

- Mejoramiento de la capacidad productiva y emprendedora de las poblaciones estudiantiles.

- Fortalecimiento de la educación para el desarrollo sostenible y una vida saludable.

Por su parte, el MINAET define en su Plan Estratégico de 2010-2015, los siguientes objetivos: "Fortalecimiento de las capacidades institucionales, que le conduzcan a adoptar decisiones y desarrollar funciones asertivas, efectivas, eficientes y sostenibles, para la consecución de sus objetivos y potestades" y como áreas estratégicas: "Conservación y uso sostenible de la biodiversidad y los recursos naturales". (Picado L. \& Quesada A, 2012: 53)

Con respecto al Ministerio de Agricultura y Ganaderia (MAG), incluye en su Plan Estratégico Nacional y en el Plan Regional de Desarrollo 2006-2010, los siguientes objetivos: "Promover y difundir la agricultura orgánica para la inserción en los mercados, mejoramiento del ambiente y la salud humana" y "Promoción e incorporación de prácticas productivas, que permitan el uso racional y la conservación de los recursos naturales" (Picado L \& Quesada A, 2012: 54).

Por consiguiente, en Costa Rica existe una intencionalidad por rescatar PS en los programas y proyectos institucionales, sin embargo es débil este propósito, dado que sólo tres de las instituciones investigadas lo tiene visibilizado de manera explícita, mientras que las demás no lo tienen contemplado. Situación que limita trabajar y profundizar en su implementación en el país.

\subsection{2) A pesar del reconocimiento político-gerencial del enfoque, a nivel operativo} se prioriza la aplicación de otros enfoques

En lo referente a los resultados sobre condiciones en los ambientes internos que favorecen o limitan el fortalecimiento del enfoque, para las investigadoras determinan que: si bien, a nivel interno tienen explícitos como prioridad de actuación el enfoque de Promoción de la Salud, en sus procesos de planificación, no siempre se cumple con lo establecido en el marco legal, los análisis de situación de salud y los planes estratégicos, pues en la práctica se establecen otras prioridades. Así nos lo reforzó dos funcionarios entrevistados: 
“...si la encargada de Promoción de la Salud se pensiona o no está, se detiene el proceso" (MS)

“...prevalece la visión biologista del ser humano en los servicios de salud, hay ausencia de normativa que defina la organización, el quehacer y la competencia institucional en Promoción de la Salud, así como insuficientes recursos técnicos, programáticos y financieros"(CCSS).

\subsection{3) ¿El enfoque de Promoción de la Salud: un mito o una realidad en los servicios} sociales de la salud?

Una de las grandes sorpresas identificadas en el proceso de investigación evaluativa fue que las personas entrevistadas desconocían el tipo de servicios que presta la institución, además desconocían si estos servicios incorporaban el enfoque de Promoción de la Salud.

Un elemento que se resaltó en todas las respuestas de las personas entrevistadas, fue la identificación de programas, proyectos y actividades de la Prevención y Atención de la Enfermedad, como proyectos de Promoción de la Salud. Por ejemplo, varios de los entrevistados rescataban Programas de Salud Escolar, Programa de Acción de Promotores Recreativos y Comisión de Salud y Alimentación Local, Proyectos Deportivos, con Proyectos de Manejo de Residuos, Proyecto de Conservación de Cuencas Hídricas, Proyectos de Estilos de Vida Saludables (risoterapia, alimentación saludable, relaciones saludables, actividad física, sexualidad sana), entre otros.

De las personas entrevistadas, las que manifiestan un poco de claridad del tipo proyectos desde la perspectiva de Promoción de la Salud fueron de la Caja Costarricense de Seguro Social y del Ministerio de Educación Públicas.

Otro elemento que se destaca en las respuestas fue que los proyectos denominados de Promoción de la Salud se dirigen más a ciertos grupos poblacionales y áreas temáticas. Entre los grupos poblacionales a los que más se dirigen estos proyectos son: niños, niñas, adultos mayores, población con discapacidad, mujeres líderes y funcionarios internos de las instituciones. Con respecto a las áreas temáticas están: medio ambiente, alimentación, culturales y deportivos. 


\subsection{El perfil de aptitudes caracterizado por las personas entrevistadas}

Un elemento fundamental para el desempeño de las funciones en PS, es que se tenga definido un perfil de aptitudes que oriente las acciones y esfuerzos de funcionarias y funcionarios hacia la consecución de los objetivos en este campo, por tal motivo, un interés de las investigadoras fue conocer las características de quienes ejercen dichas funciones en las instituciones en estudio.

3.6.1) Reconocimiento de las responsabilidades individuales, para ejercer sus funciones, pero limitadas por las políticas institucionales

Al respecto las personas entrevistadas logran reconocer un alto sentido de responsabilidad de los funcionarios y las funcionarias, para incorporar PS en su quehacer profesional, pero se reconoce las limitaciones institucionales para estimular este esfuerzo o interés.

En este sentido, funcionarios del Ministerio de Salud y de la Universidad de Costa Rica coincidieron que no les ha resultado fácil realizar las respectivas acciones. Esto se refleja en las siguientes respuestas: “...no ha sido fácil desarrollar estas acciones y se necesita mucha constancia y no dejarse quebrar por los retos que implica romper con paradigmas..." (MS).

"Porque en el tema de promoción, debe haber como mucha sensibilidad, agrega, la gente participa porque se sabe que el efecto es positivo" (UCR).

Igualmente los entrevistados destacan que tienen iniciativa, capacidad para tomar decisiones, creatividad, liderazgo. No obstante, también refieren que el ejercicio de las mismas, se ve limitada por las directrices políticas institucionales, en donde existen estructura lineal normativa indicativa, así lo señala un entrevistado: "el problema con la toma de decisiones es que siempre existe la limitante si se cuenta o no se cuenta con el aval de las jefaturas para desarrollar eso que se está decidiendo." (CCSS)

Por las expresiones obtenidas, reflejan una ambivalencia, porque por un lado existe un reconocimiento e interés del personal acerca de la importancia de ejecutar la PS, no obstante, al parecer hay falta de apoyo y compromiso de las jefaturas institucionales para que esto se lleva a cabo, quienes establecen otras prioridades en la parte de prevención y atención de la enfermedad. 
3.6.2) Falta de claridad conceptual referente a innovación y asertividad en la mayoría de personal entrevistado e igualmente limitaciones para su desarrollo

Otras características significativas para el desempeño en PS, son la innovación y creatividad. Referente a estos dos conceptos se identificó falta de claridad en las personas entrevistadas, en cuanto no lograron conceptualizarlo y no diferenciarlo el uno del otro.

A continuación algunos ejemplos:

"... desde que se forman grupos hay asertividad" (jefatura local)" y con respecto a la innovación se reconoce que: "Para PS no se podría pensar en alguien que no tenga innovación. Tiene que ser capaz de innovar,...". (Director Regional del MS). Mientras las jefaturas locales expresan: “...existen limitaciones para innovar como: tener que ejecutar acciones muy directivas, multiplicidad de funciones...", "se le dice que eso no está dentro de sus funciones..."

A pesar de la falta de claridad, los entrevistados si reconocen lo importante de estas características aptitudinales de los profesionales, para realizar acciones en PS.

3.6.3) Habilidades sociales en el cumplimiento de las funciones, pero con debilidades técnicas para su desarrollo

Hay opiniones encontradas en lo referente a la participación positiva de los funcionarios en los equipos de trabajo, pues mientras unos responden que si hay integración positiva, otros mencionan que se dificulta, dado que ya sean funcionarios o miembros de comunidades tienen otras prioridades.

Las mayores fortalezas en este tema lo constituye la comunicación, tanto verbal como escrita, “...por las características de la profesión no pueden carecer de dicha habilidad..." (MEP-UCR), asimismo la empatía, "...que tratan de ponerse en el lugar de las personas o compañeros con quienes tienen que trabajar para poder apoyar y desarrollar su trabajo..." (MS)

Una debilidad presente en este tipo de habilidades, es el carácter individualista que tiene las personas miembros de las instituciones, así lo manifestó un entrevistado: "una habilidad, si lo vemos de manera individual, es que la gente 
toma decisiones personalmente, pero dentro de la institución hay cosas que no se pueden, entonces tiene que consultarse" (CCSS)

3.6.4) Las capacidades gerenciales constituyen las mayores debilidades presentes en el personal

Las personas entrevistadas no lograron identificar con claridad que es el liderazgo situacional. Por ejemplo, algunas jefaturas locales señalan, "cambia según la jefatura". Otros se refirieren a liderazgo participativo. En el caso de direcciones regionales del Ministerio de Salud destacan que "... se está trabajando en ello sobre todo los gerentes, quienes según indicación institucional deben ejercerlo..."

Consecuentemente con las limitaciones anotadas en las características individuales, algunas jefaturas locales exponen que lo existente es más bien el liderazgo autocrático.

En relación con el desarrollo del personal, lo asocian más al área de capacitaciones, la planificación con programación y las alianzas con coordinaciones. Por tal motivo, denota una debilidad en cuanto al manejo de estos conceptos y herramientas gerenciales.

Por otro lado, los grupos entrevistados hacen referencias a las habilidades que constituyen fortalezas en el personal, tales como: negociación, concertación y resolución de conflictos, con la salvedad que las mismas están más desarrollados en algunos funcionarios y funcionarias que en otros.

También señalan limitaciones para el desarrollo de habilidades relacionadas con capacitaciones y conducción de procesos de planificación, por las siguientes razones: las altas demandas de trabajo institucional exigen priorizar las opciones de capacitación y además, que asistir a las mismas dependen de la voluntad de la jefatura en turno, dado que unos las facilitan y otros no. Por último, la falta de seguimiento y evaluación de los procesos de planificación.

\subsection{Existe una capacidad institucional limitada e insuficiente}

Otra categoría de evaluación que se consideró importante fue la capacidad instalada con que cuentan las instituciones para ejecutar los servicios sociales con enfoque en Promoción de la Salud. 
Un hallazgo que se obtuvo de los tres grupos de actores sociales entrevistados, es que las diversas instituciones disponen de presupuestos, recursos de infraestructura, soporte técnico y apoyo logístico, pero se caracteriza de la siguiente manera:

1) El presupuesto institucional se distribuye en las múltiples labores que ejerce, por lo que no existe un presupuesto específico para las acciones con Enfoque de Promoción de la Salud, sino que se compiten con los otros tipos de servicios que se brinda a la institución. Por lo general, los presupuestos se destinan para subsidiar gastos de alimentación, documentación, implementos de oficina, entre otros.

El pago de personal para hacer actividades, como por ejemplo promotores de actividad física, tiene que ser gestionado por los mismos miembros de las comunidades. Ejemplo de ello son los proyectos de actividad física y recreación de la CCSS y del ICODER, cuyos grupos deben financiar los respectivos instructores.

2) Todas las instituciones cuentan con salas de reunión para actividades de capacitación o encuentros de coordinación con actores sociales, pero no cuentan con infraestructura especial para realizar actividades deportivas o de reunión masiva. La mayoría de estas actividades se realiza en las instalaciones existentes en la comunidad, como gimnasios, canchas, aulas de escuelas, colegios, universidades y salones comunales. Para los funcionarios de la CCSS, tal situación se convierte en una oportunidad, dado que permite que las actividades con enfoque en Promoción de la Salud se realice en contextos externos al área hospitalaria o clínica, que se caracterizan por ser ambientes más enfermizos que de salud.

Algunos funcionarios de las instituciones públicas gestionan los espacios recreativos que cuentan sus respectivas instituciones, pero tienen la limitante que varios de estos lugares se ubican en zonas alejadas del contexto de la población meta, lo que genera mayores gastos para la ejecución de actividades grupales o comunales.

3) Un aspecto que resaltan con todas las respuestas de las personas entrevistadas, es que en los últimos años las instituciones públicas se han preocupado por modernizar los equipos tecnológicos, de comunicación y audiovisual, tales como: computadoras, proyectores, faxes, entre otros. La mayoría cuenta con acceso a internet.

Palobra No. 13. Agosto de 2013 
Este hallazgo se convierte en una fortaleza de las diversas instancias institucionales; el problema radica en que estos materiales y equipos resultan insuficientes para la demanda. No son materiales exclusivos para los servicios sociales con enfoque en Promoción de la Salud.

4) Una condición que comparten todas las instituciones con respecto al apoyo logístico, es que los funcionarios a cargo de la ejecución de servicios sociales en el campo de la salud tiene como recargo la realización de acciones administrativas y de secretaría, limitando la ejecución de sus actividades medulares en las instituciones.

A raíz de los procesos de modernización y control institucional, las acciones y actividades administrativas han aumentado considerablemente, lo que se convierte en un recargo para los y las funcionarias que ejecutan servicios de Promoción de la Salud.

En cuanto a las limitaciones se determinan: los programas están centralizados, así como los presupuestos por lo que deben realizar un gran trámite para gestionar los recursos requeridos por el nivel local. Para realizar algunas actividades deben acudir a empresa privada u otras instancias porque no cuentan con dicho apoyo.

\subsection{Las alianzas estratégicas y articulaciones de actores sociales: una oportunidad para fomentar y fortalecer el enfoque en la región}

A partir de los resultados reflejados en el estudio se evidencia que en la Región Central de Occidente existen procesos de participación social y comunal que constituyen una gran oportunidad de integración de alianzas y articulaciones de actores sociales para el fomento de la Promoción de la Salud. En este sentido, se destacan las siguientes acciones de articulación intersectorial: promoción de estilos de vida saludable, se encuentran las siguientes instituciones: MS, MEP, CCSS, ICODER, Comités de Deportes, UCR.

Otras instituciones, como MS, MINAET, MAG, Instituto de Desarrollo Agrario (IDA), Acueductos y Alcantarillados (AYA), municipalidades, población civil, se articulan en torno al abordaje de situaciones relacionadas con el ambiente (trapiches ecológicos, sembradores de tomate, horticultura, proyecto educación ambiental, proyectos de agroindustria, proyecto protección de la vida silvestre-ahorro del agua- protección de mantos acuíferos). También 
resaltan la participación de los siguientes grupos: pequeños y medianos productores y jóvenes rurales, jóvenes empresariales, comunidades educativas, personas con discapacidad, adultos mayores, ASADAS, grupos de mujeres, comunidades y comisiones intersectoriales.

Una limitante que resaltan algunos entrevistados con referencia a las alianzas estratégicas es la injerencia y pluralidad de partidos políticos, dado que dificultan el consenso y negociación en relación con algunos proyectos locales.

\section{CONCLUSIONES GENERALES}

A nivel internacional, nacional e institucional se reconoce la importancia de los enfoques de Promoción de la Salud, Prevención y Atención de la Enfermedad, en el abordaje del proceso salud-enfermedad, dando especial énfasis al fomento de la Promoción de la Salud. No obstante, ello queda en una intencionalidad, puesto que operativamente se continúan privilegiando los enfoques de Prevención y Atención.

Las leyes y decretos específicos que han sido catalogados como los afines a la Promoción de la Salud, en Costa Rica, están promulgados desde una lógica preventiva, lo que refuerza que los servicios sociales de la salud estipulen sus objetivos y grupos poblacionales desde la perspectiva de riesgo y focalización.

En cuanto a los planes estratégicos nacionales y algunos regionales de las instituciones en estudio, se encuentra que solamente los correspondientes al MS, CCSS, la UCR, y el ICODER, consideran de manera explícita la Promoción de la Salud, no obstante en la práctica, como se mencionó anteriormente, privilegian el abordaje a factores de riesgo y atención de daños.

El denominador común de las experiencias desarrolladas, en el campo de la Promoción de la Salud, son algunas actividades correspondientes a Estilos de Vida Saludables, predominando actividad física, alimentación saludable y ambientes saludables, por lo que se dejan de lado otros factores protectores de la salud (cultura de la paz, potencialidades individuales y colectivas, relaciones interpersonales, proyectos de vida, políticas públicas saludables, entre otros) y desarrollo humano, condiciones de vida y políticas públicas.

Si bien se reconocen una serie de fortalezas en las habilidades individuales, sociales, gerenciales, así como en competencias técnicas, se determinan 
también debilidades. Además se manifiesta falta de claridad de algunos conceptos básicos de estas habilidades y destrezas, que afectan el desempeño del personal en el cumplimiento de sus funciones.

En cuanto a la capacidad instalada, se pudo constatar que existe una serie de recursos institucionales, pero resultan insuficientes para atender sus demandas y en particular las de Promoción de la Salud.

Existe una gran oportunidad para impulsar y fortalecer el enfoque de PS, cual es, la amplia representación y funcionamiento de instituciones, organizaciones y grupos, que ya han formado alianzas estrategias.

Con el análisis de los diferentes hallazgos identificados en la investigación evaluativa, se recomienda: hacer una revisión del marco legal para que la fundamentación de las mismas sean consecuente con la lógica en PS, favorecer las directrices y la capacidad instalada de las instituciones que permitan fortalecer las acciones en PS, elaborar un perfil de aptitudes que oriente las funciones profesionales en PS y se mantengan procesos de capacitación continua a los funcionarios de las instituciones sobre este tema.

\section{REFERENCIAS BIBLIOGRÁFICAS}

Bergonzoli, G. (1994) Evolución epistemológica de la salud. En: Bergonzoli, G.y D. Victoria (eds.). Rectoría y vigilancia de la salud. San José, OPS/OMS, 1994

Caja Costarricense del Seguro Social. (2007 a) Plan Estratégico de Promoción de la Salud 2008-2012. Dirección de Desarrollo de Servicios de Salud. Área Colectiva, Promoción de la Salud. CCSS, Costa Rica.

Caja Costarricense del Seguro Social (2007 b). Dirección de Desarrollo de Servicios de Salud. Área Colectiva, Promoción de la Salud. Plan Estratégico de Promoción de la Salud 2008-2012. CCSS, Costa Rica.

Caja Costarricense del Seguro Social (2007 c). Políticas Institucionales. San José Costa Rica.

Carmona L., Rozo C. \& Mogollon A. (2005) La salud y la promoción de la salud: una aproximación a su desarrollo histórico y social. En: Revista Ciencias de la Salud. Vol. 3. Número 11. Universidad de Rosario. 
Comisión Regional Intersectorial de Promoción de la Salud (2008) Plan Regional Intersectorial de Promoción de la Salud, 2008-2011. Comisión Regional Intersectorial de Promoción de la Salud. San Ramón, Alajuela,

Gómez, M (2004) Evaluación de servicios sociales. Pedagogía Social y de Trabajo Social. Editorial Gedisa. Biblioteca de Educación. España.

Ministerio de Educación Pública (Marzo, 2009) Guía para la elaboración del Plan Operativo Anual 2010. Concepciones básicas. Dirección de Planificación Institucional Departamento de Programación y Evaluación. San José, Costa Rica.

Ministerio de Salud (2008-2010 a). Plan Estratégico Institucional 2008-2010. Dirección General de la Salud.

Ministerio de Salud (2007 b) Políticas institucionales. San José, Costa Rica.

Ministerio de Agricultura y Ganadería (2006) Plan Regional de Desarrollo del Sector Productivo 20062010 Comité Sectorial Regional Agropecuario, Región Central Occidental.

Morera, N (2001) La gerencia de las organizaciones productoras de servicios sociales. Editorial de la Universidad de Costa Rica. Costa Rica.

Picado L \& Quesada A. ( 2012) Informe de los resultados de la investigación "La Promoción de la Salud en el contexto de las instituciones públicas de la Región Central Occidental de Costa Rica. Coordinación de Investigación, Sede de Occidente, UCR y Dirección Regional de Rectoría de la Salud Central Occidente, Ministerio de Salud. 